\title{
Effects of Surface Contaminants on Interface Dipole Formation of 8-hydroxyquinolinolato-lithium Layer
}

\author{
Received 21 December, 2021; accepted 4 January, 2022
}

\begin{abstract}
Hyunbok Lee*(i)
Department of Physics and Institute of Quantum Convergence Technology, Kangwon National University, Chuncheon 24341, Republic of Korea
\end{abstract}

\section{*Corresponding author E-mail: hyunbok@kangwon.ac.kr}

\begin{abstract}
To obtain efficient organic devices, minimizing the charge injection barrier between the metal electrode and organic layer is of great importance. For this, a fundamental understanding of the formation mechanism of the interface dipole that changes the work function of the electrodes is necessary. In this study, different formations of the interface dipole of 8-hydroxyquinolinolato-lithium (Liq) on clean and contaminated metal surfaces was investigated using in situ ultraviolet photoelectron spectroscopy. On a clean Au surface, Liq induced a large interface dipole, and the work function significantly decreased, similar to other conducting electrodes. However, on a contaminated Ag surface, a negligible interface dipole was formed with the deposition of the Liq layer; thus, the work function hardly changed. This was caused by the decoupling between the electron wave functions of Liq and $\mathrm{Ag}$ by the contamination layer. Thus, to efficiently tailor the work function using a charge injection layer, the surface conditions of the electrodes should be carefully considered.
\end{abstract}

Keywords: 8-hydroxyquinolinolato-lithium, Energy level alignment, Interface dipole, Organic semiconductor, Ultraviolet photoelectron spectroscopy

\section{Introduction}

An efficient energy level alignment is essential to achieve high performance of organic devices [1-5]. This means that the Fermi level $\left(E_{\mathrm{F}}\right)$ of an electrode should match the charge transport level of an organic semiconductor. However, in general, the $E_{\mathrm{F}}$ of neat electrodes is not well aligned to either the lowest unoccupied molecular orbital (LUMO, electron transport level) or the highest occupied molecular orbital (HOMO, hole transport level) of organic semiconductors. Thus, the work function of the electrodes should be modified. To this end, various hole/electron injection layers have been employed to increase/ decrease the work function of electrodes. These charge injection layers induce an interface dipole on the electrode surface, thereby changing the work function. However, a fundamental understanding of the formation mechanism of the interface dipole remains insufficient. Thus, unveiling the origin of the interface dipole is of great importance in choosing an efficient charge injection layer.

One of the most popularly used electron injection layers for lowering the work function of electrodes in organic light-emitting diodes and organic photovoltaic cells is 8-hydroxyquinolinolato-lithium (Liq) [6-9]. Liq significantly improves electron injection, similar to LiF. However, Liq also has the advantage of a much lower evaporation temperature than LiF. To understand the origin of enhanced electron injection, interfacial electronic structures of Liq and metals have been investigated [10-13]. According to these previous studies, electron transfer from Liq to metal occurs, and a large interface dipole is formed by charge redistribution. As a result, the work function of electrodes is significantly reduced, which is beneficial for cathode use. However, the degree of electron transfer can vary according to the condition of the metal surface. For example, Grobosch et al. [14] reported that the magnitude of the interface dipole at $\alpha$-sexithiophene/metal contacts is considerably lower on contaminated metal surfaces than on clean metal surfaces. However, the effects of surface contaminants on the interface dipole formation of Liq are not yet well understood. If metal is exposed to air after deposition, C- and O-based contaminants inevitably exist on the surface. Thus, the work function may not be efficiently decreased with the Liq layer on the contaminated metal surface.

In this study, different formations of the interface dipole of Liq on clean $\mathrm{Au}$ and contaminated $\mathrm{Ag}$ metal surfaces were investigated using in situ ultraviolet photoelectron spectroscopy (UPS) measurements. An accurate analysis of the electronic structure at the organic/metal interface can be performed only by in situ UPS experiments [15]. By comparing the interface dipoles formed on two different surfaces, the origin of the work function reduction using the Liq layer is discussed.

\section{Experimental details}

Two electrodes with different surface conditions were prepared: an Au plate was cleaned in the vacuum using Ar ion sputtering, and Ag foil was used without cleaning. Liq was purchased from SigmaAldrich (St. Louis, MO, USA). The electronic structures of Liq on clean $\mathrm{Au}$ and contaminated Ag were determined in a stepwise manner using the in situ UPS system, which was composed of deposition and analysis chambers connected via a gate valve. Liq was degassed sufficiently in the deposition chamber before the main experiments. Afterward, the Liq layer was deposited at the rate of $0.01 \mathrm{~nm} \mathrm{~s}^{-1}$ via 
thermal evaporation using a Knudsen cell. The deposition rate and total thickness of the Liq layer were monitored using a quartz crystal microbalance. The thickness of the Liq layer increased until the spectral changes were saturated. The analysis chamber was equipped with a $\mathrm{He} \mathrm{I}_{\alpha}(h v=21.22 \mathrm{eV})$ ultraviolet discharge lamp and a PHI $5700 \mathrm{spec}-$ trometer. The base pressures of the deposition and analysis chambers were approximately $10^{-8}$ and $10^{-9}$ Torr, respectively. At each deposition step, the sample was transferred to the analysis chamber using a magnetic transfer arm without breaking the vacuum; then, the UPS spectrum was recorded. To determine the secondary electron cutoff (SEC) of the UPS spectra, a sample bias of $-15 \mathrm{~V}$ was applied.

\section{Results and discussion}

Figure 1 shows the (a) SEC and (b) HOMO regions of the in situ UPS spectra of Liq $(0,0.2,1.6,2.6$, and $4.0 \mathrm{~nm})$ on cleaned Au. To clearly show the work function shift with an increase in the thickness of the Liq layer, the SEC region spectra were normalized and drawn with a kinetic energy scale. In the HOMO region, the Shirley-type background was removed from the measured HOMO region spectrum. The $E_{\mathrm{F}}$ edge of $\mathrm{Au}$ was observed at $0 \mathrm{eV}$, and a spectral broadening of $0.25 \mathrm{eV}$ was observed. In the current study, to reliably determine the energy position, this thermal and instrumental broadening was applied to the SEC and HOMO onset. The detailed procedure was described elsewhere [16]. In Fig. 1(a), the work function of Au was measured as $5.07 \mathrm{eV}$. As the 0.2 -nm-thick Liq layer was deposited, the SEC shifted toward a lower kinetic energy by $1.02 \mathrm{eV}$. This means that the work function significantly decreased with the deposition of the ultrathin Liq layer. With Liq layers thicker than $0.2 \mathrm{~nm}$, the work function decreased more. For the 1.6-, 2.6-, and 4.0-nm-thick Liq layers, the work function was measured as $3.79,3.74$, and $3.66 \mathrm{eV}$, respectively. Thus, the work function of 4.0-nm-thick Liq on Au was lower by 1.41 $\mathrm{eV}$ than that of clean $\mathrm{Au}$. This indicates that the work function of clean Au can be dramatically reduced with the deposition of the Liq layer.

In Fig. 1(b), the Au 5d valence features are clearly observed in the bottommost spectrum of clean Au. Because the spectral intensity of $\mathrm{Au}$ was quite higher than that of Liq on $\mathrm{Au}$, the clean $\mathrm{Au}$ spectrum was minimized for clarity. As the Liq layer was deposited, the HOMO features of Liq emerged near $2 \mathrm{eV}$. However, the HOMO onset of Liq could not be reliably determined at the 0.2 -nm thickness owing to its too-low coverage. Thus, the HOMO onset of Liq was determined with thicknesses thicker than $0.2 \mathrm{~nm}$. With the $1.6-\mathrm{nm}$ thickness of the Liq layer, the HOMO onset was observed at $1.57 \mathrm{eV}$. With an increase in the thickness of the Liq layer, the HOMO onset shifted toward a higher binding energy. A HOMO onset at the final deposition step of $4.0 \mathrm{~nm}$ was observed at $1.74 \mathrm{eV}$. This HOMO level shift implies the band bending of the Liq layer on clean Au.

Figure 2 shows the (a) SEC and (b) HOMO regions of the in situ UPS spectra of Liq $(0,0.4,0.8,1.8$, and $2.8 \mathrm{~nm})$ on contaminated Ag. In Fig. 2(a), the work function of the contaminated Ag was measured as $4.31 \mathrm{eV}$. As the $0.4-\mathrm{nm}$-thick Liq layer was deposited, the SEC shifted toward a higher kinetic energy by $0.39 \mathrm{eV}$. This increase in the work function with partial coverage of the Liq layer is a contrary phenomenon to the clean Au case (Fig. 1) and those of other conducting substrates reported previously [13]. The origin of the increased work function by the partially covered Liq layer is not clear at present, and further study is necessary. However, one possible explanation pertains to changes in the molecular orientation [17]. Different molecular orientations induce different dipole moments, which can alter the direction of the work function shift. However, with Liq layers thicker than $0.4 \mathrm{~nm}$, the work function monotonically decreases, as the Liq layer on

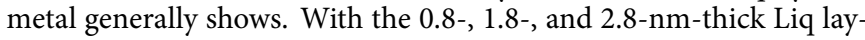
ers, the work function was measured as $4.45,4.42$, and $4.18 \mathrm{eV}$. Thus, during the deposition of the 2.8-nm-thick Liq layer, the total shift in the work function was measured as only $0.13 \mathrm{eV}$.

In Fig. 2(b), in the bottommost spectrum of contaminated Ag, the $E_{\mathrm{F}}$ edge was not observed because the Ag surface was covered by Cand O-based contaminants. Additionally, $\mathrm{O} 2 \mathrm{p}$ features near $3 \mathrm{eV}$ were clearly observed, also showing that the Ag surface was contaminated [18]. As the Liq layer was deposited, the HOMO features of Liq were observed at approximately $1.5 \mathrm{eV}$. Like the Liq/clean Au case, the reliable HOMO onset of Liq was determined at thicknesses greater than $0.4 \mathrm{~nm}$. With the $0.8-\mathrm{nm}$-thick Liq layer, the HOMO onset of Liq was observed at $0.95 \mathrm{eV}$. With an increase in the thickness of the Liq layer, the HOMO onset gradually shifted toward a higher binding energy. With the 1.8- and 2.8-nm-thick Liq layers, HOMO onsets were observed at 1.04 and $1.10 \mathrm{eV}$. Thus, the HOMO level shift was 0.15 $\mathrm{eV}$ during the deposition of the 2.8-nm-thick Liq layer, implying that band bending occurred in the Liq layer on contaminated Ag.

Figure 3 shows the energy level alignment of (a) Liq/clean $\mathrm{Au}$ and (b) Liq/contaminated Ag. The LUMO level of Liq was estimated using the band gap $(3.00 \mathrm{eV})$ determined by X-ray absorption and emission spectroscopy measurements [19]. In both cases, the ionization energy of the thick Liq layer was almost the same within the experimental error margin ( 5.28 and $5.40 \mathrm{eV}$, respectively). Band bending was evaluated by the HOMO level shift, whereas the interface dipole was calculated as the difference between the SEC shift and band bending [20]. In Fig. 3(a), the work function of clean Au was $5.07 \mathrm{eV}$. With the de-

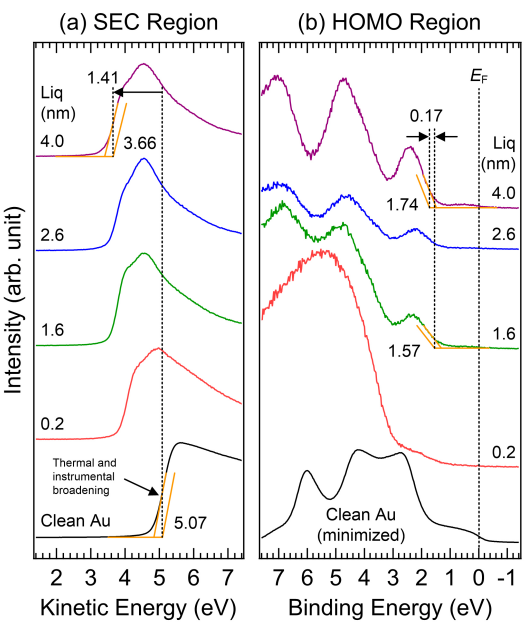

Figure 1. In situ UPS spectra of the (a) SEC and (b) HOMO regions of Liq (0, 0.2, 1.6, 2.6, and $4.0 \mathrm{~nm}) /$ clean $\mathrm{Au}$.

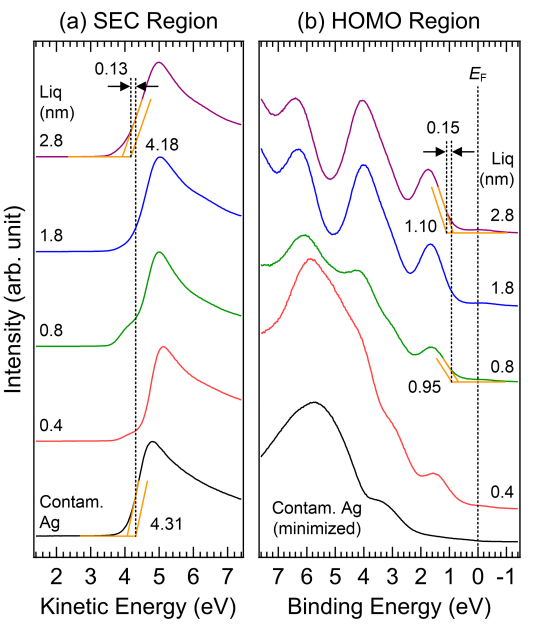

Figure 2. In situ UPS spectra of the (a) SEC and (b) HOMO regions of Liq (0, 0.4, $0.8,1.8$, and $2.8 \mathrm{~nm}) /$ contaminated $\mathrm{Ag}$. 
(a) Liq on clean $\mathrm{Au}$

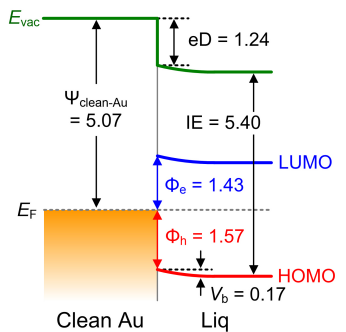

(b) Liq on contaminated $\mathrm{Ag}$

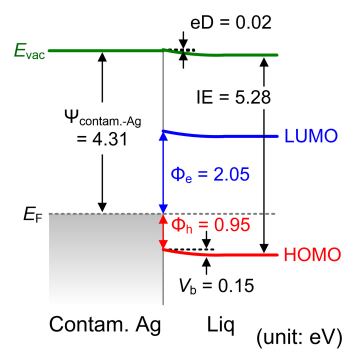

Figure 3. Energy level diagrams of Liq on (a) clean $A u$ and (b) contaminated $A g$ $\Psi, E_{\mathrm{VAC}}, E_{\mathrm{F}}, V_{\mathrm{b}}, \mathrm{DD}, \mathrm{IE}, \boldsymbol{\Phi}, \Phi_{\mathrm{b}}$ LUMO and HOMO denote the work function, vacuum level, Fermi level, band bending, interface dipole, ionization energy, elec tron injection layer, hole injection layer, lowest unoccupied molecular orbital, and highest occupied molecular orbital, respectively (unit: eV).

position of the Liq layer, the work function was significantly reduced to $3.83 \mathrm{eV}$, owing to the formation of the interface dipole of $1.24 \mathrm{eV}$. The electron and hole injection barriers from clean Au to Liq were 1.43 and $1.57 \mathrm{eV}$, respectively. The band bending in the Liq layer was 0.17 $\mathrm{eV}$.

However, in Fig. 3(b), the energy level alignment of Liq on contaminated $\mathrm{Ag}$ was quite different from that of Liq on clean Au. The work function of the contaminated Ag was $4.31 \mathrm{eV}$. Although the Liq layer was deposited, the vacuum level hardly shifted. In other words, only a negligible interface dipole $(0.02 \mathrm{eV})$ formed on contaminated Ag. As a result, the electron and hole injection barriers were 2.05 and 0.95 $\mathrm{eV}$, respectively. Such a low hole injection barrier and a vacuum level alignment with the Liq layer have not been reported with other metal contacts. By this time, interface dipoles larger than $1 \mathrm{eV}$ were observed in Liq on conducting substrates $[10,11,13]$. Thus, this marginal interface dipole of Liq on contaminated Ag might have caused unexpected results.

However, such different formations of the interface dipole were also observed in other organic/metal contacts. Wan et al. [21] reported that a large interface dipole was formed at the $N, N^{\prime}$-bis-(1naphthyl)- $N, N^{\prime}$-diphenyl-1,1'-biphenyl-4,4'-diamine (NPB)/clean Au contact, whereas a much smaller interface dipole was formed at the $\mathrm{NPB} /$ contaminated Au contact. Similarly, the formation of a smaller dipole on the contaminated metal surface was observed in rubrene on $\mathrm{Ag}$ [22]. The origin of these smaller dipoles would be the decoupling of the organic layer and metal surface by the contamination layer. When the Liq layer is deposited on the metal surface, the electron wave functions of Liq and metal overlap, and electron transfer occurs from Liq to metal to achieve thermal equilibrium. As a result, a large interface dipole is formed. However, when C- and O-based contaminants exist on the metal surface, the overlap between the electron wave functions of Liq and metal is significantly suppressed. Thus, electron transfer from Liq to metal is considerably hindered, and only a small interface dipole is formed. This is the origin of the different formations of the interface dipole on clean $\mathrm{Au}$ and contaminated Ag. These results indicate that work function modification using the Liq layer should be performed upon careful consideration of the surface conditions of the electrodes.

\section{Conclusions}

In this study, the effects of surface contaminants on the interface dipole formation of Liq were investigated via in situ UPS analysis. On a clean Au surface, the Liq layer formed a significantly large interface dipole $(1.24 \mathrm{eV})$ via electron transfer from Liq to Au. However, on the contaminated Ag surface, the Liq layer formed a negligible interface dipole $(0.02 \mathrm{eV})$, and the vacuum level was almost aligned. This difference can be attributed to the $\mathrm{C}$ - and O-based contamination layer decoupling the electron wave functions of Liq and Ag. Thus, electron transfer was significantly interrupted. These results suggest that work function reductions using the Liq layer cannot be efficiently obtained on air-exposed electrodes. This study provides fundamental information for achieving an efficient energy level alignment in organic devices using the charge injection layer.

\section{Acknowledgements}

This study was supported by the National Research Foundation of Korea (NRF- 2021R1A2C100932 and 2018R1A6A1A03025582).

\section{Conflict of Interest}

The authors declare no conflicts of interest.

\section{ORCID}

Hyunbok Lee

https://orcid.org/0000-0002-3046-1524

\section{References}

[1] H. Ishii, K. Sugiyama, E. Ito, and K. Seki, Adv. Mater. 11, 605 (1999).

[2] A. Kahn, N. Koch, and W. Gao, J. Polym. Sci. B-Polym. Phys. 41, 2529 (2003).

[3] N. Koch, ChemPhysChem 8, 1438 (2007).

[4] H. Lee, S. W. Cho, and Y. Yi, Curr. Appl. Phys. 16, 1533 (2016).

[5] S. Olthof, Adv. Opt. Mater. 9, 2100227 (2021).

[6] C. Schmitz, H.-W. Schmidt, and M. Thelakkat, Chem. Mater. 12, $3012(2000)$.

[7] Y. Lee, J. Kim, S. Kwon, C.-K. Min, Y. Yi, J. W. Kim, B. Koo, and M. Hong, Org. Electron. 9, 407 (2008).

[8] X. Zheng, Y. Wu, R. Sun, W. Zhu, X. Jiang, Z. Zhang, and S. Xu, Thin Solid Films 478, 252 (2005).

[9] Y. Zou, Z. Deng, L. Yu, H. Du, Y. Yin, M. Zhou, and Y. Wang, Thin Solid Films 520, 5811 (2012).

[10] K. Cho, S. W. Cho, P. E. Jeon, H. Lee, C.-N. Whang, K. Jeong, S. J. Kang, and Y. Yi, Appl. Phys. Lett. 92, 093304 (2008).

[11] K. Cho, S. W. Cho, P. E. Jeon, H. Lee, C.-N. Whang, K. Jeong, S. J. Kang, and Y. Yi, Synth. Met. 158, 984 (2008).

[12] Y. Yi, Y. M. Lee, Y. Park, and J. W. Kim, Phys. Chem. Chem. Phys. 12, 9441 (2010).

[13] S. Park, Y. Yi, S. W. Cho, and H. Lee, Chem. Phys. Lett. 652, 102 (2016).

[14] M. Grobosch and M. Knupfer, Adv. Mater. 19, 754 (2007).

[15] H. Lüth, Solid Surfaces, Interfaces and Thin Films, 6th ed. (Springer-Verlag, Berlin, 2015).

[16] Y. Lee, H. Lee, S. Park, and Y. Yi, Appl. Phys. Lett. 101, 233305 (2012).

[17] S. Kera, Y. Yabuuchi, H. Yamane, H. Setoyama, K. K. Okudaira, A. Kahn, and N. Ueno, Phys. Rev. B 70, 085304 (2004).

[18] T. C. R. Rocha, A. Oestereich, D. V. Demidov, M. Hävecker, S. Zafeiratos, G. Weinberg, V. I. Bukhtiyarov, A. Knop-Gericke, and R. Schlögl, Phys. Chem. Chem. Phys. 14, 4554 (2012).

[19] Y. K. Park, B. K. Ryu, J. Kim, S. Yeo, C. S. Oh, S. W. Cho, H. Lee, and H. Ogasawara, Curr. Appl. Phys. 30, 91 (2021).

[20] R. Schlaf, B. A. Parkinson, P. A. Lee, K. W. Nebesny, and N. R. Armstrong, J. Phys. Chem. B 103, 2984 (1999).

[21] A. Wan, J. Hwang, F. Amy, and A. Kahn, Org. Electron. 6, 47 (2005).

[22] S. Sinha, C.-H. Wang, and M. Mukherjee, Appl. Surf. Sci. 409, 22 (2017) 